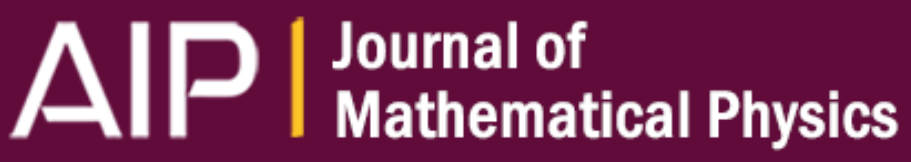

Quasi-Hopf algebras and representations of octonions and other quasialgebras

Florin Panaite and Freddy Van Oystaeyen

Citation: Journal of Mathematical Physics 45, 3912 (2004); doi: 10.1063/1.1789280

View online: http://dx.doi.org/10.1063/1.1789280

View Table of Contents: http://scitation.aip.org/content/aip/journal/jmp/45/10?ver=pdfcov

Published by the AIP Publishing

\section{AlP Re-register for Table of Content Alerts}




\title{
Quasi-Hopf algebras and representations of octonions and other quasialgebras
}

\author{
Florin Panaite ${ }^{a)}$ \\ Institute of Mathematics of the Romanian Academy, P.O. Box 1-764, \\ RO-70700 Bucharest, Romania \\ Freddy Van Oystaeyen ${ }^{\text {b) }}$ \\ Department of Mathematics and Computer Science, University of Antwerp, \\ Middelheimlaan 1, B-2020 Antwerp, Belgium
}

(Received 29 January 2004; accepted 7 June 2004; published 12 October 2004)

\begin{abstract}
Modules over a quasialgebra (here, by quasialgebra we mean a left $H$-module algebra, where $H$ is a quasi-Hopf algebra), as defined by Albuquerque and Majid, coincide with modules over a certain associative algebra, a quasi-Hopf smash product. As a consequence of this, we get that the category of modules over the octonions is isomorphic to the category of modules over the algebra of $8 \times 8$ real matrices. We provide a new approach to the endomorphism quasialgebra associated to a left $H$-module, which in the finite dimensional case yields the same results as the one of Albuquerque and Majid. We discuss possible definitions as endomorphism quasialgebras for Heisenberg doubles of a finite dimensional quasi-Hopf algebra. (C) 2004 American Institute of Physics. [DOI: 10.1063/1.1789280]
\end{abstract}

\section{INTRODUCTION}

The starting point of this paper was the following question we asked ourselves: What could be a module over the octonions? The usual approach to octonions (we refer to Ref. 2 for a recent survey of the existing theory for the octonions) does not provide an answer. However, it turned out that such an answer is possible using the recent Hopf-algebraic approach to octonions proposed by Albuquerque and Majid in Ref. 1. Namely, they first introduced the concept of a "quasialgebra," as being an algebra in a tensor category (in this paper we restrict the term to algebras in the tensor category of modules over a quasi-Hopf algebra) and then proved that the (nonassociative) algebra of octonions is such a quasialgebra; they defined also a module over a quasialgebra $A$ as being an $A$-module in the same tensor category where $A$ lives as an algebra and hence a suitable definition of modules over the octonions may be derived from this.

If $H$ is a quasi-Hopf algebra and $M$ is a finite dimensional left $H$-module, Albuquerque and Majid constructed the so-called "endomorphism quasialgebra" of $M$-which is built on the vector space $\operatorname{End}(M)$ and will be denoted below by end $(M)$ —using "tensor-categorical" techniques, that is by introducing first a quasialgebra structure on $M \otimes M^{*}$ and then transfering it to $\operatorname{End}(M)$ via the linear isomorphism $\operatorname{End}(M) \simeq M \otimes M^{*}$. Also, if $A$ is an algebra in the category of left $H$-modules, they proved that setting a structure of an $A$-module on $M$ is equivalent to giving a quasialgebra map $A \rightarrow \operatorname{end}(M)$.

There is an obvious analogy with what happens for other classes of algebras (associative or Lie), where setting a module structure on a vector space is equivalent to giving an algebra map from the algebra to a certain "endomorphism algebra" constructed out of the vector space, but in those cases one can construct the "endomorphism algebra" of any object (not necessarily finite dimensional), so it is natural to see whether this is possible also for the class of quasialgebras.

\footnotetext{
${ }^{a)}$ Electronic mail: florin.panaite@imar.ro

${ }^{b}$ Electronic mail: francine.schoeters@ua.ac.be
} 
In order to explain our contribution to this subject, let us first recall some results from Ref. 6. First, if $H$ is a quasi-Hopf algebra, $B$ an associative algebra and $v: H \rightarrow B$ an algebra map, then on $B$ one can introduce a structure of an algebra in the tensor category of left $H$-modules (a left $H$-module algebra structure, in the terminology of Ref. 6), denoted by $B^{v}$. Second, if $A$ is a left $H$-module algebra, an associative algebra, denoted by $A \# H$ and called the smash product, has been introduced in Ref. 6 and the left $A \# H$-modules were described. These turned out to coincide with the $A$-modules in the category of left $H$-modules (called left $A, H$-modules in Ref. 6).

The first aim of this paper is to point out that the modules over a quasialgebra, as defined by Albuquerque and Majid, coincide with modules over an associative algebra, namely, a quasi-Hopf smash product. As a consequence, we obtain that the category of modules over a twisted group quasialgebra $k G_{F}$ is isomorphic to the category of modules over the algebra of $|G|$ by $|G|$ matrices over $k$ (where $|G|$ is the order of the group $G$ ), and in particular that the category of modules over the octonions is isomorphic to the category of modules over the algebra of $8 \times 8$ real matrices.

The second aim is to provide a different approach to the endomorphism quasialgebra associated to an object, which works also in the infinite dimensional case. Namely, if $H$ is a quasi-Hopf algebra, $A$ a left $H$-module algebra and $M$ a left $H$-module, we have the algebra map $v: H$ $\rightarrow \operatorname{End}(M), v(h)(m)=h \cdot m$, so we can consider the left $H$-module algebra $\operatorname{End}(M)^{v}$ (this will be the endomorphism quasialgebra associated to $M$ ); then we prove that setting a structure of a left $A$, $H$-module on $M$ is equivalent to giving a morphism of left $H$-module algebras $\varphi: A \rightarrow \operatorname{End}(M)^{v}$ (the proof relies on the use of the smash product $A \# H$ ). Also, we prove that if $M$ is a finite dimensional left $H$-module, then $\operatorname{End}(M)^{v}$ coincides with end $(M)$ defined in Ref. 1 as left $H$-module algebras, and that if $M$ is moreover a left $A, H$-module, then the map $\varphi$ coincides with the similar map $A \rightarrow \operatorname{end}(M)$ defined by Albuquerque and Majid.

As an immediate consequence of the identification $\operatorname{End}(M)^{v} \equiv \operatorname{end}(M)$ and of results in Ref. 4, we obtain that if $M$ is a finite dimensional Yetter-Drinfeld $H$-module, then $\operatorname{End}(M)^{v}$ is a YetterDrinfeld $H$-module algebra.

The analogy with the classes of associative or Lie algebras mentioned before can be pushed forward. Namely, the concepts of a bimodule over an associative or Lie algebra are a manifestation of a single concept, that of a bimodule over an algebra with respect to a class of algebras (see Ref. 18). We prove that if $H$ is a quasi-bialgebra and $A$ is a left $H$-module algebra, then an $A$-bimodule with respect to the class of left $H$-module algebras, in the sense of Ref. 18, is the same as an $A$-bimodule in the tensor category of left $H$-modules.

In the final section we discuss possible definitions as endomorphism quasialgebras for Heisenberg doubles of a finite dimensional quasi-Hopf algebra.

\section{PRELIMINARIES}

We work over a commutative field $k$. All algebras, linear spaces, etc., will be over $k$; unadorned $\otimes$ means $\otimes_{k}$. Following Drinfeld, ${ }^{9}$ a quasi-bialgebra is a fourtuple $(H, \Delta, \varepsilon, \Phi)$, where $H$ is an associative algebra with unit, $\Phi$ is an invertible element in $H \otimes H \otimes H$, and $\Delta: H \rightarrow H \otimes H$ and $\varepsilon: H \rightarrow k$ are algebra homomorphisms satisfying the identities

$$
(\mathrm{id} \otimes \Delta)(\Delta(h))=\Phi(\Delta \otimes \mathrm{id})(\Delta(h)) \Phi^{-1},
$$

$$
(\mathrm{id} \otimes \varepsilon)(\Delta(h))=h \otimes 1, \quad(\varepsilon \otimes \mathrm{id})(\Delta(h))=1 \otimes h,
$$

for all $h \in H$, and $\Phi$ has to be a normalized 3-cocycle, in the sense that

$$
(1 \otimes \Phi)(\mathrm{id} \otimes \Delta \otimes \mathrm{id})(\Phi)(\Phi \otimes 1)=(\mathrm{id} \otimes \mathrm{id} \otimes \Delta)(\Phi)(\Delta \otimes \mathrm{id} \otimes \mathrm{id})(\Phi)
$$




$$
(\mathrm{id} \otimes \varepsilon \otimes \mathrm{id})(\Phi)=1 \otimes 1 \otimes 1 .
$$

The map $\Delta$ is called the coproduct or the comultiplication, $\varepsilon$ the counit and $\Phi$ the reassociator. As for Hopf algebras ${ }^{19}$ we denote $\Delta(h)=\Sigma h_{1} \otimes h_{2}$, but since $\Delta$ is only quasi-coassociative we adopt the further convention

$$
(\Delta \otimes \mathrm{id})(\Delta(h))=\sum h_{(1,1)} \otimes h_{(1,2)} \otimes h_{2}, \quad(\mathrm{id} \otimes \Delta)(\Delta(h))=\sum h_{1} \otimes h_{(2,1)} \otimes h_{(2,2)},
$$

for all $h \in H$. We will denote the tensor components of $\Phi$ by capital letters, and those of $\Phi^{-1}$ by small letters, namely,

$$
\begin{gathered}
\Phi=\sum X^{1} \otimes X^{2} \otimes X^{3}=\sum T^{1} \otimes T^{2} \otimes T^{3}=\sum V^{1} \otimes V^{2} \otimes V^{3}=\cdots \\
\Phi^{-1}=\sum x^{1} \otimes x^{2} \otimes x^{3}=\sum t^{1} \otimes t^{2} \otimes t^{3}=\sum v^{1} \otimes v^{2} \otimes v^{3}=\cdots .
\end{gathered}
$$

The quasi-bialgebra $H$ is called a quasi-Hopf algebra if there exists an antiautomorphism $S$ of the algebra $H$ and elements $\alpha, \beta \in H$ such that, for all $h \in H$, we have

$$
\begin{gathered}
\sum S\left(h_{1}\right) \alpha h_{2}=\varepsilon(h) \alpha \text { and } \sum h_{1} \beta S\left(h_{2}\right)=\varepsilon(h) \beta, \\
\sum X^{1} \beta S\left(X^{2}\right) \alpha X^{3}=1 \text { and } \sum S\left(x^{1}\right) \alpha x^{2} \beta S\left(x^{3}\right)=1 .
\end{gathered}
$$

For a quasi-Hopf algebra the antipode is determined uniquely up to a transformation $\alpha \mapsto U \alpha$, $\beta \mapsto \beta U^{-1}, S(h) \mapsto U S(h) U^{-1}$, where $U \in H$ is invertible. The axioms for a quasi-Hopf algebra imply that $\varepsilon(\alpha) \varepsilon(\beta)=1$, so, by rescaling $\alpha$ and $\beta$, we may assume without loss of generality that $\varepsilon(\alpha)=\varepsilon(\beta)=1$ and $\varepsilon \circ S=\varepsilon$. The identities (2.2)-(2.4) also imply that

$$
(\varepsilon \otimes \mathrm{id} \otimes \mathrm{id})(\Phi)=(\mathrm{id} \otimes \mathrm{id} \otimes \varepsilon)(\Phi)=1 \otimes 1 \otimes 1 .
$$

Together with a quasi-bialgebra or a quasi-Hopf algebra $H=(H, \Delta, \varepsilon, \Phi, S, \alpha, \beta)$ we also have $H^{\text {op }}$, $H^{\text {cop }}$ and $H^{\text {op,cop }}$ as quasi-bialgebras (respectively, quasi-Hopf algebras), where "op" means opposite multiplication and "cop" means opposite comultiplication. The structures are obtained by setting $\Phi_{\mathrm{op}}=\Phi^{-1}, \quad \Phi_{\mathrm{cop}}=\left(\Phi^{-1}\right)^{321}, \quad \Phi_{\mathrm{op}, \mathrm{cop}}=\Phi^{321}, \quad S_{\mathrm{op}}=S_{\mathrm{cop}}=\left(S_{\mathrm{op}, \mathrm{cop}}\right)^{-1}=S^{-1}, \quad \alpha_{\mathrm{op}}=S^{-1}(\beta), \quad \beta_{\mathrm{op}}$ $=S^{-1}(\alpha), \alpha_{\text {cop }}=S^{-1}(\alpha), \beta_{\text {cop }}=S^{-1}(\beta), \alpha_{\text {op }, \mathrm{cop}}=\beta$, and $\beta_{\text {op }, \text { cop }}=\alpha$.

Next we recall that the definition of a quasi-bialgebra or quasi-Hopf algebra is "twist covariant" in the following sense. An invertible element $F \in H \otimes H$ is called a gauge transformation or twist if $(\varepsilon \otimes \mathrm{id})(F)=(\mathrm{id} \otimes \varepsilon)(F)=1$. If $H$ is a quasi-bialgebra or a quasi-Hopf algebra and $F$ $=\Sigma F^{1} \otimes F^{2} \in H \otimes H$ is a gauge transformation with inverse $F^{-1}=\Sigma G^{1} \otimes G^{2}$, then we can define a new quasi-bialgebra (respectively, quasi-Hopf algebra) $H_{F}$ by keeping the multiplication, unit, counit (and antipode in the case of a quasi-Hopf algebra) of $H$ and replacing the comultiplication, reassociator and the elements $\alpha$ and $\beta$ by

$$
\begin{gathered}
\Delta_{F}(h)=F \Delta(h) F^{-1}, \\
\Phi_{F}=(1 \otimes F)(\mathrm{id} \otimes \Delta)(F) \Phi(\Delta \otimes \mathrm{id})\left(F^{-1}\right)\left(F^{-1} \otimes 1\right), \\
\alpha_{F}=\sum S\left(G^{1}\right) \alpha G^{2}, \quad \beta_{F}=\sum F^{1} \beta S\left(F^{2}\right) .
\end{gathered}
$$

It is well known that the antipode of a Hopf algebra is an anti-coalgebra morphism. For a quasi-Hopf algebra, we have the following statement: there exists a gauge transformation $f \in H$ $\otimes H$ such that 


$$
f \Delta(S(h)) f^{-1}=\sum(S \otimes S)\left(\Delta^{\mathrm{cop}}(h)\right) \text { for all } h \in H .
$$

The element $f$ can be computed explicitly. First set

$$
\begin{aligned}
& \sum A^{1} \otimes A^{2} \otimes A^{3} \otimes A^{4}=(\Phi \otimes 1)(\Delta \otimes \mathrm{id} \otimes \mathrm{id})\left(\Phi^{-1}\right), \\
& \sum B^{1} \otimes B^{2} \otimes B^{3} \otimes B^{4}=(\Delta \otimes \mathrm{id} \otimes \mathrm{id})(\Phi)\left(\Phi^{-1} \otimes 1\right),
\end{aligned}
$$

and then define $\gamma, \delta \in H \otimes H$ by

$$
\gamma=\sum S\left(A^{2}\right) \alpha A^{3} \otimes S\left(A^{1}\right) \alpha A^{4} \text { and } \delta=\sum B^{1} \beta S\left(B^{4}\right) \otimes B^{2} \beta S\left(B^{3}\right) .
$$

Then $f$ and $f^{-1}$ are given by the formulas

$$
\begin{gathered}
f=\sum(S \otimes S)\left(\Delta^{\mathrm{cop}}\left(x^{1}\right)\right) \gamma \Delta\left(x^{2} \beta S\left(x^{3}\right)\right), \\
f^{-1}=\sum \Delta\left(S\left(x^{1}\right) \alpha x^{2}\right) \delta(S \otimes S)\left(\Delta^{\mathrm{cop}}\left(x^{3}\right)\right) .
\end{gathered}
$$

If $H$ is a quasi-Hopf algebra, following Refs. 10 and 11 we may define the elements

$$
\begin{aligned}
& p_{R}=\sum p^{1} \otimes p^{2}=\sum x^{1} \otimes x^{2} \beta S\left(x^{3}\right), q_{R}=\sum q^{1} \otimes q^{2}=\sum X^{1} \otimes S^{-1}\left(\alpha X^{3}\right) X^{2}, \\
& p_{L}=\sum \tilde{p}^{1} \otimes \widetilde{p}^{2}=\sum X^{2} S^{-1}\left(X^{1} \beta\right) \otimes X^{3}, q_{L}=\sum \widetilde{q}^{1} \otimes \widetilde{q}^{2}=\sum S\left(x^{1}\right) \alpha x^{2} \otimes x^{3}
\end{aligned}
$$

satisfying the relations (for all $h \in H$ ):

$$
\begin{gathered}
\sum q_{1}^{1} p^{1} \otimes q_{2}^{1} p^{2} S\left(q^{2}\right)=1 \otimes 1, \quad \sum q^{1} p_{1}^{1} \otimes S^{-1}\left(p^{2}\right) q^{2} p_{2}^{1}=1 \otimes 1, \\
\sum S\left(\tilde{p}^{1}\right) \tilde{q}^{1} \tilde{p}_{1}^{2} \otimes \widetilde{q}^{2} \widetilde{p}_{2}^{2}=1 \otimes 1, \quad \sum \widetilde{q}_{1}^{2} \tilde{p}^{1} S^{-1}\left(\widetilde{q}^{1}\right) \otimes \widetilde{q}_{2}^{2} \widetilde{p}^{2}=1 \otimes 1, \\
\sum \Delta\left(h_{1}\right) p_{R}\left[1 \otimes S\left(h_{2}\right)\right]=p_{R}[h \otimes 1], \quad \sum\left[1 \otimes S^{-1}\left(h_{2}\right)\right] q_{R} \Delta\left(h_{1}\right)=[h \otimes 1] q_{R}, \\
\sum \Delta\left(h_{2}\right) p_{L}\left[S^{-1}\left(h_{1}\right) \otimes 1\right]=p_{L}[1 \otimes h], \quad \sum\left[S\left(h_{1}\right) \otimes 1\right] q_{L} \Delta\left(h_{2}\right)=[1 \otimes h] q_{L} .
\end{gathered}
$$

Suppose that $(H, \Delta, \varepsilon, \Phi)$ is a quasi-bialgebra. If $U, V, W$ are left (right) $H$-modules, define $a_{U, V, W}$, $\mathbf{a}_{U, V, W}:(U \otimes V) \otimes W \rightarrow U \otimes(V \otimes W)$ by

$$
\begin{gathered}
a_{U, V, W}((u \otimes v) \otimes w)=\boldsymbol{\Phi} \cdot(u \otimes(v \otimes w)), \\
\mathbf{a}_{U, V, W}((u \otimes v) \otimes w)=(u \otimes(v \otimes w)) \cdot \boldsymbol{\Phi}^{-1} .
\end{gathered}
$$

The category ${ }_{H} \mathcal{M}\left(\mathcal{M}_{H}\right)$ of left (right) $H$-modules becomes a monoidal category (see Refs. 12 and 14 for the terminology) with tensor product $\otimes$ given via $\Delta$, associativity constraints $a_{U, V, W}$ $\left(\mathbf{a}_{U, V, W}\right)$, unit $k$ as a trivial $H$-module and the usual left and right unit constraints.

Now, let $H$ be a quasi-bialgebra. We say that a $k$-vector space $A$ is a left $H$-module algebra if it is an algebra in the monoidal category ${ }_{H} \mathcal{M}$, that is $A$ has a multiplication and a usual unit $1_{A}$ satisfying the following conditions:

$$
\left(a a^{\prime}\right) a^{\prime \prime}=\sum\left(X^{1} \cdot a\right)\left[\left(X^{2} \cdot a^{\prime}\right)\left(X^{3} \cdot a^{\prime \prime}\right)\right]
$$




$$
\begin{gathered}
h \cdot\left(a a^{\prime}\right)=\sum\left(h_{1} \cdot a\right)\left(h_{2} \cdot a^{\prime}\right), \\
h \cdot 1_{A}=\varepsilon(h) 1_{A},
\end{gathered}
$$

for all $a, a^{\prime}, a^{\prime \prime} \in A$ and $h \in H$, where $h \otimes a \rightarrow h \cdot a$ is the left $H$-module structure of $A$. Following Ref. 6 we define the smash product $A \# H$ as follows: as vector space $A \# H$ is $A \otimes H$ (elements $a$ $\otimes h$ will be written $a \# h$ ) with multiplication given by

$$
(a \# h)\left(a^{\prime} \# h^{\prime}\right)=\sum\left(x^{1} \cdot a\right)\left(x^{2} h_{1} \cdot a^{\prime}\right) \# x^{3} h_{2} h^{\prime},
$$

for all $a, a^{\prime} \in A, h, h^{\prime} \in H$. Then $A \# H$ is an associative algebra with unit $1_{A} \# 1$.

If $A^{\prime}$ is another left $H$-module algebra, a map $f: A \rightarrow A^{\prime}$ is a morphism of left $H$-module algebras if it is multiplicative, unital and a morphism of left $H$-modules.

For further use we need also the notion of right $H$-module algebra. Let $H$ be a quasi-bialgebra. We say that a $k$-linear space $C$ is a right $H$-module algebra if $C$ is an algebra in the monoidal category $\mathcal{M}_{H}$, i.e., $C$ has a multiplication and a usual unit $1_{C}$ satisfying the following conditions:

$$
\begin{gathered}
\left(c c^{\prime}\right) c^{\prime \prime}=\sum\left(c \cdot x^{1}\right)\left[\left(c^{\prime} \cdot x^{2}\right)\left(c^{\prime \prime} \cdot x^{3}\right)\right], \\
\left(c c^{\prime}\right) \cdot h=\sum\left(c \cdot h_{1}\right)\left(c^{\prime} \cdot h_{2}\right), \\
1_{C} \cdot h=\varepsilon(h) 1_{C},
\end{gathered}
$$

for all $c, c^{\prime}, c^{\prime \prime} \in C$ and $h \in H$, where $c \otimes h \rightarrow c \cdot h$ is the right $H$-module structure of $C$.

Let $H$ be a quasi-bialgebra, $F \in H \otimes H$ a gauge transformation and $A$ a left $H$-module algebra. Then, following Refs. 6 and 5, we can define a new multiplication on $A$, by

$$
a \diamond b=\sum\left(G^{1} \cdot a\right)\left(G^{2} \cdot b\right), \quad \forall a, b \in A,
$$

where $F^{-1}=\Sigma G^{1} \otimes G^{2}$. If we denote by $A_{F^{-1}}$ the resulting structure, then $A_{F^{-1}}$ becomes a left $H_{F}$-module algebra, with the same unit and $H$-action as for $A$, and moreover the map

$$
\lambda: A \# H \rightarrow A_{F^{-1}} \# H_{F}, \lambda(a \# h)=\sum F^{1} \cdot a \# F^{2} h,
$$

is an algebra isomorphism.

If $H$ is a quasi-Hopf algebra, $B$ an associative algebra and $v: H \rightarrow B$ an algebra map, then, following Ref. 6, we can introduce on the vector space $B$ a left $H$-module algebra structure, denoted by $B^{v}$ in what follows, for which the multiplication, unit and left $H$-action are

$$
\begin{gathered}
b \star b^{\prime}=\sum v\left(X^{1}\right) b v\left(S\left(x^{1} X^{2}\right) \alpha x^{2} X_{1}^{3}\right) b^{\prime} v\left(S\left(x^{3} X_{2}^{3}\right)\right), \quad \forall b, b^{\prime} \in B, \\
1_{B^{v}}=v(\beta), \\
h \triangleright_{v} b=\sum v\left(h_{1}\right) b v\left(S\left(h_{2}\right)\right), \quad \forall h \in H, b \in B .
\end{gathered}
$$

If $H$ is a quasi-Hopf algebra and $A$ is a left $H$-module algebra, define the following maps:

$$
j: H \rightarrow A \# H, j(h)=1 \# h, \quad \forall h \in H,
$$




$$
i_{0}: A \rightarrow A \# H, \quad i_{0}(a)=\sum x^{1} \cdot a \# x^{2} \beta S\left(x^{3}\right), \quad \forall a \in A .
$$

Then, by Ref. $6, j$ is an algebra map and $i_{0}$ is a morphism of left $H$-module algebras from $A$ to $(A \# H)^{j}$. Moreover, the following universal property of the smash product $A \# H$ holds (see Ref. 6 , Proposition 2.9): if $B$ is an associative algebra, $v: H \rightarrow B$ is an algebra map and $\varphi: A \rightarrow B^{v}$ is a morphism of left $H$-module algebras, then there exists a unique algebra map $\varphi \# v: A \# H \rightarrow B$ such that $(\varphi \# v) \circ i_{0}=\varphi$ and $(\varphi \# v) \circ j=v$; this map may be described explicitly as follows:

$$
(\varphi \# v)(a \# h)=\sum v\left(X^{1}\right) \varphi(a) v\left(S\left(X^{2}\right) \alpha X^{3} h\right), \quad \forall a \in A, h \in H .
$$

Now recall from Ref. 6 the following concept.

Definition 2.1: Let $H$ be a quasi-bialgebra and $A$ a left $H$-module algebra. We say that $M$, a $k$-linear space, is a left $A, H$-module if

(i) $\quad M$ is a left $H$-module with action denoted by $h \otimes m \mapsto h \cdot m$.

(ii) $A$ acts weakly on $M$ to the left, i.e., there exists a $k$-linear map $A \otimes M \rightarrow M$, denoted by $a \otimes m \mapsto a \triangleright m$, such that $1_{A} \triangleright m=m$ for all $m \in M$.

(iii) The following compatibility relations hold:

$$
\begin{gathered}
a \triangleright(b \triangleright m)=\sum\left[\left(x^{1} \cdot a\right)\left(x^{2} \cdot b\right)\right] \triangleright\left(x^{3} \cdot m\right), \\
h \cdot(a \triangleright m)=\sum\left(h_{1} \cdot a\right) \triangleright\left(h_{2} \cdot m\right),
\end{gathered}
$$

for all $h \in H, a, b \in A$ and $m \in M$ (these conditions may be expressed equivalently by saying that $M$ is a left $A$-module in the tensor category $\left.{ }_{H} \mathcal{M}\right)$.

The category of all left $A, H$-modules, morphisms being the maps that are $H$-linear and preserve the $A$-action, will be denoted by ${ }_{A, H} \mathcal{M}$.

Let $A \# H$-mod be the category of left $A \# H$-modules; we have the following result (see Ref. 6).

Proposition 2.2: Let $H$ be a quasi-bialgebra and $A$ a left $H$-module algebra. Then the categories ${ }_{A, H} \mathcal{M}$ and $A \# H$-mod are isomorphic.

The isomorphism is given as follows. If $M \in A \# H$-mod with $A \# H$-module structure given by $(a \# h) \otimes m \mapsto(a \# h) \cdot m$, then $M \in_{A, H} \mathcal{M}$ with $A$-action given by $a \triangleright m=(a \# 1) \cdot m$ and $H$-action given by $h \cdot m=(1 \# h) \cdot m$. Conversely, if $M \in_{A, H} \mathcal{M}$, then $M$ becomes a left $A \# H$-module with action $(a \# h) \cdot m=a \triangleright(h \cdot m)$.

\section{OCTONIONS AND OTHER TWISTED GROUP QUASIALGEBRAS}

Let $G$ be a finite group, $k G$ its group algebra and $k(G)$ the dual Hopf algebra of $k G$. We recall first the setting in Ref. 1, but in a quasi-Hopf framework (not dual quasi-Hopf as in Ref. 1). Let $T: G \times G \rightarrow k^{*}$ be an invertible map, with inverse $F: G \times G \rightarrow k^{*}$, such that $T(1, x)=T(x, 1)=1$ for all $x \in G$, and regard $T$ and $F$ as extended to $k G \otimes k G$, so we can regard them as elements in $k(G) \otimes k(G)$, where they become gauge transformations. So, we have the Hopf algebra $k(G), T$ a gauge transformation on it, and we have the left $k(G)$-module algebra $k G$ [with the left regular action of $k(G)$ on $k G$, that is $p \rightarrow x=p(x) x$ for all $p \in k(G)$ and $x \in G]$. From the preliminaries, we know that we can consider the left $k(G)_{T}$-module algebra $k G_{F}$, and also that we have an algebra isomorphism,

$$
k G_{F} \# k(G)_{T} \simeq k G \# k(G) .
$$

Let us mention that the multiplication in $k G_{F}$ (denoted in Ref. 1 by $k_{F} G$ ), which is given by 


$$
x \cdot{ }_{F} y=F(x, y) x y, \quad \forall x, y \in G,
$$

is in general nonassociative, and that $k(G)_{T}$, though is a Hopf algebra (being commutative), is regarded here as a quasi-Hopf algebra, with reassociator obtained by extending the map $\phi: G$ $\times G \times G \rightarrow k^{*}$ given by

$$
\phi(x, y, z)=\frac{T(x, y z) T(y, z)}{T(x y, z) T(x, y)}=\frac{F(x, y) F(x y, z)}{F(y, z) F(x, y z)},
$$

so in general $k G_{F}$ is a $k(G)_{T}$-module algebra only in the quasi-Hopf sense (not in the Hopf sense).

Now, it is well known (see Ref. 16) that $k G \# k(G)$, which is the Heisenberg double of $k G$, is isomorphic as an algebra to the matrix algebra $M_{|G|}(k)$, where $|G|$ is the order of $G$. Since left $k G_{F}$, $k(G)_{T}$-modules coincide to left $k G_{F} \# k(G)_{T}$-modules, we get the following result.

Proposition 3.1: The category of left $k G_{F}, k(G)_{T}$-modules is isomorphic to the category of left $M_{|G|}(k)$-modules.

In particular, as proved in Ref. 1 , the algebra $\mathcal{O}$ of octonions is of the form $k G_{F}$, for $G=Z_{2}$ $\times Z_{2} \times Z_{2}$ (where $Z_{2}$ is the group with two elements) and a certain map $F$, so we get the following as a consequence.

Corollary 3.2: The category of modules over the octonions is isomorphic to the category of modules over the algebra of $8 \times 8$ real matrices.

\section{THE ENDOMORPHISM QUASIALGEBRA ASSOCIATED TO A MODULE}

Lemma 4.1: Let $H$ be a quasi-Hopf algebra, $B, C$ associative algebras, $\eta: B \rightarrow C, j: H \rightarrow B$, $v: H \rightarrow C$ algebra maps such that $\eta^{\circ} j=v$. Then the map $\eta: B^{j} \rightarrow C^{v}$ is a morphism of left H-module algebras.

Proof: Follows by a direct computation, using the formulas (2.31)-(2.33).

Let $H$ be a quasi-Hopf algebra and $M$ a left $H$-module, with action denoted by $h \otimes m \mapsto h \cdot m$. Consider the (usual) associative algebra $\operatorname{End}(M)$ (with composition) and define $v: H \rightarrow \operatorname{End}(M)$, $v(h)(m)=h \cdot m$, which is an algebra map, so we can consider the left $H$-module algebra $\operatorname{End}(M)^{v}$, whose multiplication, unit and $H$-action are given as follows [using the formulas (2.31)-(2.33)]:

$$
\begin{gathered}
\left(u \star u^{\prime}\right)(m)=\sum X^{1} \cdot u\left(S\left(x^{1} X^{2}\right) \alpha x^{2} X_{1}^{3} \cdot u^{\prime}\left(S\left(x^{3} X_{2}^{3}\right) \cdot m\right)\right), \\
1_{\operatorname{End}(M)^{v}}(m)=v(\beta)(m)=\beta \cdot m \\
\left(h \triangleright_{v} u\right)(m)=\sum h_{1} \cdot u\left(S\left(h_{2}\right) \cdot m\right)
\end{gathered}
$$

for all $h \in H, u, u^{\prime} \in \operatorname{End}(M)^{v}, m \in M$.

Suppose now that we have also a left $H$-module algebra $A$, with notation $h \otimes a \mapsto h \cdot a$ and $a$ $\otimes a^{\prime} \mapsto a a^{\prime}$. Our first aim is to prove the following result.

Theorem 4.2: Setting a structure of a left A, H-module on $M$ is equivalent to giving a morphism of left $H$-module algebras $\varphi: A \rightarrow \operatorname{End}(M)^{v}$. The correspondence is given as follows: if $M$ is a left A, H-module (with A-action denoted by $a \otimes m \mapsto a \triangleright m$ ) then the map $\varphi: A$ $\rightarrow \operatorname{End}(M)^{v}$ is given by

$$
\varphi(a)(m)=\sum\left(p^{1} \cdot a\right) \triangleright\left(p^{2} \cdot m\right), \quad \forall a \in A, m \in M,
$$

where $p_{R}=\sum p^{1} \otimes p^{2}=\sum x^{1} \otimes x^{2} \beta S\left(x^{3}\right)$. Conversely, if $\varphi: A \rightarrow \operatorname{End}(M)^{v}$ is a morphism of left $H$-module algebras, then $M$ becomes a left $A, H$-module, with A-action given by

$$
a \triangleright m=\sum q^{1} \cdot \varphi(a)\left(S\left(q^{2}\right) \cdot m\right), \quad \forall a \in A, m \in M
$$


where $q_{R}=\sum q^{1} \otimes q^{2}=\Sigma X^{1} \otimes S^{-1}\left(\alpha X^{3}\right) X^{2}$, and the H-action being the original H-module structure of $M$.

Proof: Suppose first that $M$ is a left $A, H$-module, with $A$-action $a \otimes m \mapsto a \triangleright m$. As we know, this is equivalent to $M$ being a left $A \# H$-module, with structure

$$
(a \# h) \cdot m=a \triangleright(h \cdot m), \quad \forall a \in A, h \in H, m \in M .
$$

So, considering the usual associative algebra $\operatorname{End}(M)$, we obtain an algebra map $\eta: A \# H$ $\rightarrow \operatorname{End}(M), \quad \eta(a \# h)(m)=(a \# h) \cdot m$. We also have the canonical algebra map $j: H \rightarrow A \# H, j(h)$ $=1 \# h$; since we obviously have that $\eta^{\circ} j=v$, we may apply the previous lemma and obtain that the map $\eta:(A \# H)^{j} \rightarrow \operatorname{End}(M)^{v}$ is a morphism of left $H$-module algebras. It follows from the preliminaries that the map $i_{0}: A \rightarrow(A \# H)^{j}, i_{0}(a)=\Sigma p^{1} \cdot a \# p^{2}$, where $p_{R}=\Sigma p^{1} \otimes p^{2}=\Sigma x^{1} \otimes x^{2} \beta S\left(x^{3}\right)$, is a morphism of left $H$-module algebras, so the composition

$$
\varphi=\eta \circ i_{0}: A \rightarrow \operatorname{End}(M)^{v}
$$

is also a morphism of left $H$-module algebras, and one can easily check that it is given by

$$
\varphi(a)(m)=\sum\left(p^{1} \cdot a\right) \triangleright\left(p^{2} \cdot m\right), \quad \forall a \in A, m \in M .
$$

Conversely, let $\varphi: A \rightarrow \operatorname{End}(M)^{v}$ be a morphism of left $H$-module algebras; by applying the universal property of the smash product $A \# H$ (see Sec. II) for $B=\operatorname{End}(M)$, we obtain the algebra map $\varphi \# v: A \# H \rightarrow \operatorname{End}(M)$, which [using the formula (2.36)] may be expressed as follows:

$$
(\varphi \# v)(a \# h)(m)=\sum q^{1} \cdot \varphi(a)\left(S\left(q^{2}\right) h \cdot m\right), \quad \forall a \in A, h \in H, \quad m \in M .
$$

Hence, $M$ becomes a left $A \# H$-module (i.e., a left $A, H$-module) with action

$$
(a \# h) \cdot m=\sum q^{1} \cdot \varphi(a)\left(S\left(q^{2}\right) h \cdot m\right), \quad \forall a \in A, h \in H, \quad m \in M .
$$

In particular, the $A$-action is given by

$$
a \triangleright m=(a \# 1) \cdot m=\sum q^{1} \cdot \varphi(a)\left(S\left(q^{2}\right) \cdot m\right), \quad \forall a \in A, m \in M,
$$

and, using the fact that $\Sigma q^{1} \beta S\left(q^{2}\right)=1$ [which follows from the relation (2.6)], we obtain that the $H$-action is given by

$$
(1 \# h) \cdot m=\sum q^{1} \beta S\left(q^{2}\right) h \cdot m=h \cdot m, \quad \forall h \in H, \quad m \in M .
$$

Now the only thing left to prove is that the two correspondences are inverse to each other.

If $M$ is an $A, H$-module with $A$-action denoted by $\triangleright$, if $\varphi$ is the associated map $\varphi: A$ $\rightarrow \operatorname{End}(M)^{v}$ and we denote by $\triangleright^{\prime}$ the $A$-action associated to $\varphi$, we have (for all $a \in A$ and $m$ $\in M)$ :

$$
\begin{aligned}
& a \triangleright^{\prime} m=\sum q^{1} \cdot\left(\left(p^{1} \cdot a\right) \triangleright\left(p^{2} S\left(q^{2}\right) \cdot m\right)\right) \\
& (2.38)=\sum\left(q_{1}^{1} p^{1} \cdot a\right) \triangleright\left(q_{2}^{1} p^{2} S\left(q^{2}\right) \cdot m\right) \\
& (2.19)=a \triangleright m .
\end{aligned}
$$

Conversely, if $\varphi: A \rightarrow \operatorname{End}(M)^{v}$ is a left $H$-module algebra map, if $\triangleright$ is the $A$-action obtained from $\varphi$ and $\varphi^{\prime}$ is the map obtained from this $A, H$-module structure on $M$, we have (for all $a \in A$ and $m \in M)$ 


$$
\begin{aligned}
\varphi^{\prime}(a)(m) & =\sum\left(p^{1} \cdot a\right) \triangleright\left(p^{2} \cdot m\right) \\
& =\sum q^{1} \cdot \varphi\left(p^{1} \cdot a\right)\left(S\left(q^{2}\right) p^{2} \cdot m\right) \\
& =\sum q^{1} \cdot\left(\left(p^{1} \triangleright_{v} \varphi(a)\right)\left(S\left(q^{2}\right) p^{2} \cdot m\right)\right) \\
(4.3) & =\sum q^{1} \cdot\left(p_{1}^{1} \cdot \varphi(a)\left(S\left(p_{2}^{1}\right) S\left(q^{2}\right) p^{2} \cdot m\right)\right) \\
& =\sum q^{1} p_{1}^{1} \cdot \varphi(a)\left(S\left(S^{-1}\left(p^{2}\right) q^{2} p_{2}^{1}\right) \cdot m\right) \\
(2.19) & =\varphi(a)(m),
\end{aligned}
$$

and the proof is complete.

By taking in the Theorem $A=\operatorname{End}(M)^{v}$ and $\varphi=\mathrm{id}$, we obtain the following consequence:

Corollary 4.3: If $H$ is a quasi-Hopf algebra and $M$ is a left $H$-module, then $M$ becomes a left $\operatorname{End}(M)^{v}, H$-module [i.e., a left $\operatorname{End}(M)^{v} \# H$-module], with $\operatorname{End}(M)^{v}$-action given by

$$
u \triangleright m=\sum q^{1} \cdot u\left(S\left(q^{2}\right) \cdot m\right)
$$

for all $u \in \operatorname{End}(M)^{v}, m \in M$.

We study now the behavior of the construction $\operatorname{End}(M)^{v}$ under twisting. Namely, let $H$ be a quasi-Hopf algebra, $F \in H \otimes H$ a gauge transformation and $M$ a left $H$-module. Then $M$ is also a left $H_{F}$-module, with the same $H$-action. Denote by $v: H \rightarrow \operatorname{End}(M)$ and $v_{F}: H_{F} \rightarrow \operatorname{End}(M)$ the corresponding algebra maps, and consider the $H$-module algebra $\operatorname{End}(M)^{v}$ and the $H_{F}$-module algebra $\operatorname{End}(M)^{v}$; we also consider the $H_{F^{-}}$-module algebra $\operatorname{End}(M)_{F^{-1}}^{v}$. In view of similar results concerning invariance under twisting, one might expect to have $\operatorname{End}(M)^{v_{F}} \equiv \operatorname{End}(M)_{F^{-1}}^{v}$, but in general this is not true. Nevertheless, we will prove that they are isomorphic as left $H_{F}$-module algebras.

Actually, we will prove something more general. Let $H$ be a quasi-Hopf algebra, $F \in H \otimes H$ a gauge transformation, $B$ an associative algebra, $v: H \rightarrow B$ an algebra map, which will be denoted by $v_{F}$ when considered as a map from $H_{F}$ to $B$.

Proposition 4.4: The map

$$
\psi: B_{F^{-1}}^{v} \rightarrow B^{v_{F}}, \quad \psi(b)=\sum v\left(F^{1}\right) b v\left(S\left(F^{2}\right)\right), \quad \forall b \in B,
$$

is an isomorphism of left $H_{F}$-module algebras.

Proof: The map $\psi$ is obviously bijective, with inverse given by $\psi^{-1}(b)=\Sigma v\left(G^{1}\right) b v\left(S\left(G^{2}\right)\right)$, for all $b \in B$, where $F^{-1}=\Sigma G^{1} \otimes G^{2}$. Then one checks by a direct computation that $\psi$ is a morphism of left $H_{F}$-module algebras, using the formulas for $\Delta_{F}, \Phi_{F}, \alpha_{F}, \beta_{F}$ and for the multiplications, units and actions in $B^{v_{F}}$ and $B_{F^{-1}}^{v}$.

By taking $B=\operatorname{End}(M)$, we obtain the following.

Corollary 4.5: $\operatorname{End}(M)^{v_{F}}$ and $\operatorname{End}(M)_{F^{-1}}^{v}$ are isomorphic as left $H_{F^{-}}$-module algebras.

If $H$ is a quasi-Hopf algebra, the left $H$-module algebra $H^{i d_{H}}$ was denoted in Ref. 6 by $H_{0}$; its multiplication is given by

$$
g \star h=\sum X^{1} g S\left(x^{1} X^{2}\right) \alpha x^{2} X_{1}^{3} h S\left(x^{3} X_{2}^{3}\right), \quad \forall g, h \in H,
$$

the unit is $\beta$ and the left $H$-action is $h \triangleright h^{\prime}=\sum h_{1} h^{\prime} S\left(h_{2}\right)$, for all $h, h^{\prime} \in H$. Then, from the above proposition, we get also the following consequence (by taking $B=H, v=\mathrm{id}_{H}$ in the proposition):

Corollary 4.6: $\left(H_{0}\right)_{F^{-1}} \simeq\left(H_{F}\right)_{0}$ as left $H_{F^{-}}$-module algebras, with an isomorphism given by $\psi:\left(H_{0}\right)_{F^{-1}} \rightarrow\left(H_{F}\right)_{0}, \psi(h)=\Sigma F^{1} h S\left(F^{2}\right)$ for all $h \in H$, where $F=\Sigma F^{1} \otimes F^{2}$ is a gauge transformation on $H$.

For completeness' sake and further use, we also treat the case of right $A$-modules in ${ }_{H} \mathcal{M}$. 
Let $H$ be a quasi-bialgebra and $A$ a left $H$-module algebra. If we let $A^{\text {op }}$ be $A$ with opposite multiplication, then it is easy to see that $A^{\text {op }}$ becomes a left $H^{\text {cop }}$-module algebra, with the same unit and $H$-action as for $A$.

If $H$ is a quasi-Hopf algebra, $B$ an associative algebra and $v: H \rightarrow B$ an algebra map, consider $v$ as algebra map from $H^{\text {cop }}$ to $B$ and denote it by $v_{\text {cop }}$; then we have the left $H^{\text {cop }}$-module algebra

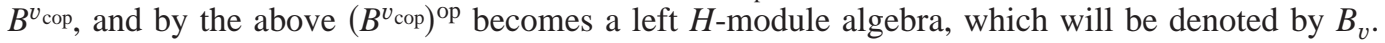
Its multiplication, unit and $H$-action are

$$
\begin{gathered}
b \cdot b^{\prime}=\sum v\left(x^{3}\right) b^{\prime} v\left(S^{-1}\left(\alpha X^{3} x^{2}\right) X^{2} x_{2}^{1}\right) b v\left(S^{-1}\left(X^{1} x_{1}^{1}\right)\right), \quad \forall b, b^{\prime} \in B, \\
1_{B_{v}}=v\left(S^{-1}(\beta)\right), \\
h \rightarrow b=\sum v\left(h_{2}\right) b v\left(S^{-1}\left(h_{1}\right)\right), \quad \forall h \in H, b \in B .
\end{gathered}
$$

Consequently, if $M$ is a left $H$-module and $v: H \rightarrow \operatorname{End}(M)$ is the algebra map as before, we can consider the left $H$-module algebra $\operatorname{End}(M)_{v}$, whose structure is

$$
\begin{gathered}
\left(u \cdot u^{\prime}\right)(m)=\sum x^{3} \cdot u^{\prime}\left(S^{-1}\left(\alpha X^{3} x^{2}\right) X^{2} x_{2}^{1} \cdot u\left(S^{-1}\left(X^{1} x_{1}^{1}\right) \cdot m\right)\right) \\
1_{\operatorname{End}(M)_{v}}(m)=S^{-1}(\beta) \cdot m \\
(h \rightarrow u)(m)=\sum h_{2} \cdot u\left(S^{-1}\left(h_{1}\right) \cdot m\right)
\end{gathered}
$$

for all $u, u^{\prime} \in \operatorname{End}(M)_{v}, m \in M, h \in H$.

Now, if $H$ is a quasi-bialgebra and $A$ is a left $H$-module algebra, we can define a right $A$, $H$-module $M$ as being a right $A$-module in ${ }_{H} \mathcal{M}$. That is, $M$ must be a left $H$-module, together with a right $A$-action $M \otimes A \rightarrow M, m \otimes a \mapsto m \triangleleft a$, with $m \triangleleft 1=m$ for all $m \in M$, such that

$$
\begin{gathered}
(m \triangleleft a) \triangleleft b=\sum\left(X^{1} \cdot m\right) \triangleleft\left[\left(X^{2} \cdot a\right)\left(X^{3} \cdot b\right)\right], \\
h \cdot(m \triangleleft a)=\sum\left(h_{1} \cdot m\right) \triangleleft\left(h_{2} \cdot a\right),
\end{gathered}
$$

for all $h \in H, m \in M, a b \in A$.

It is easy to see that $M$ being a right $A, H$-module is equivalent to $M$ being a left $A^{\text {op }}$, $H^{\mathrm{cop}}$-module (i.e., a left $A^{\mathrm{op}} \# H^{\mathrm{cop}}$-module). Then, if $H$ is moreover a quasi-Hopf algebra and using also the fact that the elements $p_{R}$ and $q_{R}$ for $H^{\text {cop }}$ may be expressed as $p_{R}^{\text {cop }}=p_{L}^{21}$ and $q_{R}^{\text {cop }}=q_{L}^{21}$,

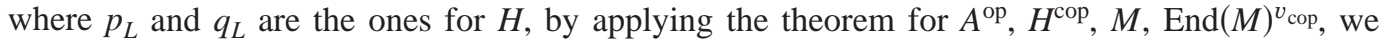
obtain the following result.

Proposition 4.7: Let $H$ be a quasi-Hopf algebra, A a left H-module algebra and $M$ a left $H$-module. Then setting a structure of a right $A, H$-module on $M$ is equivalent to giving a morphism of left $H$-module algebras $\eta: A \rightarrow \operatorname{End}(M)_{v}$. The correspondence is given as follows: if $M$ is a right $A$, H-module (with A-action denoted by $m \otimes a \mapsto m \triangleleft a$ ) then the map $\eta: A$ $\rightarrow \operatorname{End}(M)_{v}$ is given by

$$
\eta(a)(m)=\sum\left(\tilde{p}^{1} \cdot m\right) \triangleleft\left(\tilde{p}^{2} \cdot a\right), \quad \forall a \in A, \quad m \in M,
$$

where $p_{L}=\Sigma \tilde{p}^{1} \otimes \widetilde{p}^{2}=\Sigma X^{2} S^{-1}\left(X^{1} \beta\right) \otimes X^{3}$. Conversely, if $\eta: A \rightarrow \operatorname{End}(M)_{v}$ is a morphism of left $H$-module algebras, then $M$ becomes a right $A, H$-module, with $A$-action given by

$$
m \triangleleft a=\sum \widetilde{q}^{2} \cdot \eta(a)\left(S^{-1}\left(\widetilde{q}^{1}\right) \cdot m\right), \quad \forall a \in A, m \in M,
$$


where $q_{L}=\Sigma \widetilde{q}^{1} \otimes \widetilde{q}^{2}=\Sigma S\left(x^{1}\right) \alpha x^{2} \otimes x^{3}$, and the H-action being the original H-module structure of $M$.

As we have seen, the construction of $\operatorname{End}(M)_{v}$ was obtained from the one of $\operatorname{End}(M)^{v}$ by taking opposites and co-opposites. However, there is also another relation between the two constructions. Namely, if $M$ is a left $H$-module, it is well known that on the linear dual of $M$ we can introduce two left $H$-module structures, denoted by $M^{*}$ and ${ }^{*} M$, with $H$-actions $\left(h \cdot m^{*}\right)(m)$ $=m^{*}(S(h) \cdot m)$, for all $h \in H, m \in M, m^{*} \in M^{*}$, respectively, $(h * m)(m)=* m\left(S^{-1}(h) \cdot m\right)$ for all $h \in H, m \in M, * m \in * M$ [the corresponding algebra maps $H \rightarrow \operatorname{End}\left(M^{*}\right)$ and $H \rightarrow \operatorname{End}\left({ }^{*} M\right)$ are denoted by $v^{*}$, respectively, $\left.{ }^{*} v\right]$.

If $u: M \rightarrow M$ is a linear map, we denote by $u^{*}$ its transpose given by $u^{*}(\xi)=\xi^{\circ} u$ for all $\xi$ $\in M^{*}$.

We have then the following result, which generalizes a part of Proposition 4.7 in Ref. 8 .

Proposition 4.8: Let $H$ be a quasi-Hopf algebra and $M$ a left $H$-module. Then the map $u \mapsto u^{*}$ gives $H$-module algebra morphisms $\operatorname{End}(M)^{v} \rightarrow \operatorname{End}\left(M^{*}\right)_{v^{*}}$ and $\operatorname{End}(M)_{v} \rightarrow \operatorname{End}\left({ }^{*} M\right)^{* v}$, which are isomorphisms if $M$ is finite dimensional.

Proof: Follows by a direct computation, using the formulas for the structures of the $H$-module algebras $\operatorname{End}(M)_{v}$, $\operatorname{End}\left(M^{*}\right)_{v^{*}}$, etc.

Let again $H$ be a quasi-bialgebra and $A$ a left $H$-module algebra. We introduce now the concept of an $A, H$-bimodule, as being an $A$-bimodule in ${ }_{H} \mathcal{M}$. That is, $M$ must be a left $H$-module which is a left and a right $A, H$-module (with $A$-actions denoted by $\triangleright$ and $\triangleleft$ ) and such that

$$
(a \triangleright m) \triangleleft b=\sum\left(X^{1} \cdot a\right) \triangleright\left[\left(X^{2} \cdot m\right) \triangleleft\left(X^{3} \cdot b\right)\right], \quad \forall a, b \in A, m \in M .
$$

Recall now from Ref. 18 the following concept. Let $\mathcal{C}$ be a class of (not necessarily associative) algebras, $A \in \mathcal{C}$ and $M$ a linear space with two linear actions $a \otimes m \mapsto a \triangleright m$ and $m \otimes a \mapsto m \triangleleft a$ of $A$ on $M$. Then on the direct sum $A \oplus M$ one can introduce an algebra structure (called the semidirect sum or split null extension) by defining a multiplication in $A \oplus M$ by

$$
(a+m)\left(a^{\prime}+m^{\prime}\right)=a a^{\prime}+\left(m \triangleleft a^{\prime}+a \triangleright m^{\prime}\right),
$$

for all $a, a^{\prime} \in A$ and $m, m^{\prime} \in M$. Then, if $A \oplus M$ with this algebra structure is in $\mathcal{C}$, we say that $M$ is an $A$-bimodule with respect to $\mathcal{C}$. If $\mathcal{C}$ is the class of all associative algebras or of all Lie algebras, we obtain the usual concepts of bimodule for these types of algebras. We have then the following result.

Proposition 4.9: Let $H$ be a quasi-bialgebra, A a left $H$-module algebra and $M$ a k-linear space. Then $M$ is an A-bimodule with respect to the class of left $H$-module algebras (that is, A $\oplus M$ is a left $H$-module algebra) if and only if $M$ is an $A, H$-bimodule.

Proof: If $M$ is an $A, H$-bimodule, one can prove by a direct computation that $A \oplus M$ becomes a left $H$-module algebra. Conversely, if $A \oplus M$ is a left $H$-module algebra, then first $M$ becomes a left $H$-module with structure induced from the one of $A \oplus M$. Then, if the unit of $A \oplus M$ is an element of the form $a_{0}+m_{0}$, writing down the unit condition we obtain first that $a_{0}$ should be $1_{A}$, then that $1_{A} \triangleright m=m \triangleleft 1_{A}=m$ for all $m \in M$ and finally that $m_{0}=0$. Similarly, writing down the conditions for $A \oplus M$ to be a left $H$-module algebra, by taking properly particular values of the elements involved we obtain finally the conditions expressing the fact that $M$ is an $A$, $H$-bimodule.

\section{THE FINITE DIMENSIONAL CASE}

Let $H$ be a quasi-Hopf algebra, $A$ a left $H$-module algebra and $M$ a finite dimensional left $H$-module. Then, by using the identification $\operatorname{End}(M) \simeq M \otimes M^{*}$, Albuquerque and Majid introduced in Ref. 1 a left $H$-module algebra structure on $\operatorname{End}(M)$, and, if $M$ is also a left $A, H$-module, a morphism of left $H$-module algebras $A \rightarrow \operatorname{End}(M)$. Our aim now is to prove that they actually coincide with $\operatorname{End}(M)^{v}$ and $\varphi: A \rightarrow \operatorname{End}(M)^{v}$, respectively (notation as in the preceding section). 
Let us recall some well-known facts, cf. Refs. 12 and 14. If $H$ is a quasi-Hopf algebra and $M$ is a left $H$-module, we consider the two left $H$-modules $M^{*}$ (the left dual) and $* M$ (the right dual) as before. If $M$ is moreover finite dimensional and we fix $\left\{e_{1}, \ldots, e_{n}\right\}$ a basis in $M$ with $\left\{e^{1}, \ldots, e^{n}\right\}$ its dual basis in $M^{*}$, then we have the $k$-linear maps,

$$
\begin{gathered}
e v_{M}: M * \otimes M \rightarrow k, \operatorname{coev}_{M}: k \rightarrow M \otimes M *, \\
e v_{M}\left(e^{i} \otimes e_{j}\right)=e^{i}\left(\alpha \cdot e_{j}\right), \operatorname{coev}_{M}(1)=\sum_{i=1}^{n} \beta \cdot e_{i} \otimes e^{i}, \\
e v_{M}^{\prime}: M \otimes * M \rightarrow k, \operatorname{coev}_{M}^{\prime}: k \rightarrow * M \otimes M, \\
e v_{M}^{\prime}\left(e_{i} \otimes e^{j}\right)=e^{j}\left(S^{-1}(\alpha) \cdot e_{i}\right), \operatorname{coev}_{M}^{\prime}(1)=\sum_{i=1}^{n} e^{i} \otimes S^{-1}(\beta) \cdot e_{i}
\end{gathered}
$$

(the evaluation and coevaluation maps), which are $H$-linear and make $M$ a rigid object in ${ }_{H} \mathcal{M}$.

With this notation, let us recall from Ref. 1 that $M \otimes M^{*}$ becomes a left $H$-module algebra, as follows: the left $H$-module structure is the tensor product of $M$ and $M^{*}$, and the multiplication is given by the formula

$$
\left(\mathrm{id}_{M} \otimes\left(e v_{M} \otimes \mathrm{id}_{M^{*}}\right)\right) \circ\left(\mathrm{id}_{M} \otimes a_{M^{*}, M, M^{*}}^{-1}\right) \circ a_{M, M^{*}, M \otimes M^{*}}
$$

[as a map $\left(M \otimes M^{*}\right) \otimes\left(M \otimes M^{*}\right) \rightarrow M \otimes M^{*}$ ]. On the other hand, the map

$$
\lambda: \operatorname{End}(M) \rightarrow M \otimes M^{*}, \quad \lambda(u)=\sum_{i=1}^{n} u\left(e_{i}\right) \otimes e^{i}, \quad \forall u \in \operatorname{End}(M)
$$

is a linear isomorphism, with inverse

$$
\lambda^{-1}: M \otimes M^{*} \rightarrow \operatorname{End}(M), \lambda^{-1}(m \otimes \xi)\left(m^{\prime}\right)=\xi\left(m^{\prime}\right) m, \quad \forall m, m^{\prime} \in M, \quad \xi \in M^{*},
$$

so we can transfer the $H$-module algebra structure of $M \otimes M^{*}$ to $\operatorname{End}(M)$ via $\lambda$; let us denote by $\operatorname{end}(M)$ this $H$-module algebra structure on $\operatorname{End}(M)$ (this is the one appearing, with different notation, in Ref. 1).

Proposition 5.1: With notation as above, $\operatorname{end}(M)$ and $\operatorname{End}(M)^{v}$ coincide as left H-module algebras.

Proof: The fact that end $(M)=\operatorname{End}(M)^{v}$ as left $H$-modules follows from the fact, proved in Ref. 7 , that the map $\lambda$ given above, considered as a map $\lambda: \operatorname{End}(M)^{v} \rightarrow M \otimes M^{*}$, is $H$-linear. So, we only have to prove that the multiplications of $\operatorname{end}(M)$ and $\operatorname{End}(M)^{v}$ coincide. Let us compute the multiplication in end $(M)$. By using the identification end $(M) \simeq M \otimes M^{*}$ via $\lambda$ and by considering the multiplication as a map end $(M) \otimes \operatorname{end}(M) \rightarrow M \otimes M^{*}$, the sequence of compositions in formula (5.5) looks as follows [for $\left.u u^{\prime} \in \operatorname{end}(M)\right]$ :

$$
\begin{aligned}
u \otimes u^{\prime} & \mapsto \sum\left(u\left(e_{i}\right) \otimes e^{i}\right) \otimes\left(u^{\prime}\left(e_{j}\right) \otimes e^{j}\right) \\
& \mapsto \sum X^{1} \cdot u\left(e_{i}\right) \otimes\left(X^{2} \cdot e^{i} \otimes\left(X_{1}^{3} \cdot u^{\prime}\left(e_{j}\right) \otimes X_{2}^{3} \cdot e^{j}\right)\right) \\
& \mapsto \sum X^{1} \cdot u\left(e_{i}\right) \otimes\left(\left(x^{1} X^{2} \cdot e^{i} \otimes x^{2} X_{1}^{3} \cdot u^{\prime}\left(e_{j}\right)\right) \otimes x^{3} X_{2}^{3} \cdot e^{j}\right) \\
& \mapsto \sum\left(x^{1} X^{2} \cdot e^{i}\right)\left(\alpha x^{2} X_{1}^{3} \cdot u^{\prime}\left(e_{j}\right)\right) X^{1} \cdot u\left(e_{i}\right) \otimes x^{3} X_{2}^{3} \cdot e^{j} \\
& =\sum e^{i}\left(S\left(x^{1} X^{2}\right) \alpha x^{2} X_{1}^{3} \cdot u^{\prime}\left(e_{j}\right)\right) X^{1} \cdot u\left(e_{i}\right) \otimes x^{3} X_{2}^{3} \cdot e^{j}
\end{aligned}
$$




$$
=\sum X^{1} \cdot u\left(S\left(x^{1} X^{2}\right) \alpha x^{2} X_{1}^{3} \cdot u^{\prime}\left(e_{j}\right)\right) \otimes x^{3} X_{2}^{3} \cdot e^{j},
$$

so, if $m \in M$, the multiplication of end $(M)$ is

$$
\begin{aligned}
\left(u \cdot u^{\prime}\right)(m) & =\sum\left(x^{3} X_{2}^{3} \cdot e^{j}\right)(m) X^{1} \cdot u\left(S\left(x^{1} X^{2}\right) \alpha x^{2} X_{1}^{3} \cdot u^{\prime}\left(e_{j}\right)\right) \\
& =\sum e^{j}\left(S\left(x^{3} X_{2}^{3}\right) \cdot m\right) X^{1} \cdot u\left(S\left(x^{1} X^{2}\right) \alpha x^{2} X_{1}^{3} \cdot u^{\prime}\left(e_{j}\right)\right) \\
& =\sum X^{1} \cdot u\left(S\left(x^{1} X^{2}\right) \alpha x^{2} X_{1}^{3} \cdot u^{\prime}\left(S\left(x^{3} X_{2}^{3}\right) \cdot m\right)\right),
\end{aligned}
$$

and one can see that this is exactly the multiplication of $\operatorname{End}(M)^{v}$. Moreover, the unit of $\operatorname{End}(M)^{v}$ coincides with $\operatorname{coev}_{M}(1)$ after the identification $\operatorname{End}(M)^{v} \equiv M \otimes M^{*}$ via $\lambda$.

Suppose now that $M$ is not only a left $H$-module, but also a left $A, H$-module, where $A$ is a left $H$-module algebra. Then Albuquerque and Majid constructed in Ref. 1 a map $\rho: A \rightarrow \operatorname{end}(M)$, which is a morphism of left $H$-module algebras, and which is given as follows:

$$
\rho=\lambda^{-1} \circ\left(\triangleright \otimes \mathrm{id}_{M^{*}}\right) \circ a_{A, M, M^{*}}^{-1} \circ\left(\mathrm{id} \otimes \operatorname{coev}_{M}\right) .
$$

Proposition 5.2: The left $H$-module algebra maps $\varphi: A \rightarrow \operatorname{End}(M)^{v}$ and $\rho: A \rightarrow \operatorname{end}(M)$ coincide.

Proof: Let us describe the map $\rho$ explicitly. For $a \in A$, we have

$$
\begin{aligned}
\rho(a) & =\lambda^{-1} \circ\left(\triangleright \otimes \operatorname{id}_{M^{*}}\right) \circ a_{A, M, M^{*}}^{-1}\left(a \otimes\left(\sum \beta \cdot e_{i} \otimes e^{i}\right)\right) \\
& =\sum \lambda^{-1} \circ\left(\triangleright \otimes \operatorname{id}_{M^{*}}\right)\left(\left(x^{1} \cdot a \otimes x^{2} \beta \cdot e_{i}\right) \otimes x^{3} \cdot e^{i}\right) \\
& =\sum \lambda^{-1}\left(\left(x^{1} \cdot a\right) \triangleright\left(x^{2} \beta \cdot e_{i}\right) \otimes x^{3} \cdot e^{i}\right),
\end{aligned}
$$

so, for $m \in M$, we obtain

$$
\begin{aligned}
\rho(a)(m) & =\sum\left(x^{3} \cdot e^{i}\right)(m)\left(x^{1} \cdot a\right) \triangleright\left(x^{2} \beta \cdot e_{i}\right) \\
& =\sum e^{i}\left(S\left(x^{3}\right) \cdot m\right)\left(x^{1} \cdot a\right) \triangleright\left(x^{2} \beta \cdot e_{i}\right) \\
& =\sum\left(x^{1} \cdot a\right) \triangleright\left(x^{2} \beta S\left(x^{3}\right) \cdot m\right)=\sum\left(p^{1} \cdot a\right) \triangleright\left(p^{2} \cdot m\right),
\end{aligned}
$$

and this is exactly the formula for $\varphi(a)(m)$, hence we have $\rho=\varphi$.

Moreover, Albuquerque and Majid proved that if $H$ is a quasi-Hopf algebra and $M$ is a finite dimensional left $H$-module, then we have an action of end $(M)$ on $M$, which, using the identification $\operatorname{end}(M) \simeq M \otimes M^{*}$ via $\lambda$, is given by the map

$$
\left(\mathrm{id}_{M} \otimes e v_{M}\right) \circ a_{M, M^{*}, M}:\left(M \otimes M^{*}\right) \otimes M \rightarrow M .
$$

Then, using the formulas for $\lambda, e v_{M}$ and $a_{M, M^{*}, M}$, one can verify that this action coincides with the one given by the formula (4.6).

Suppose again that $H$ is a quasi-Hopf algebra and $M$ is a finite dimensional left $H$-module. Then, similarly to the definition of end $(M)$, we can define another left $H$-module algebra structure on $\operatorname{End}(M)$, by first introducing one on $* M \otimes M$ by the formula

$$
\left(\left(\mathrm{id}_{*_{M}} \otimes e v_{M}^{\prime}\right) \otimes \mathrm{id}_{M}\right) \circ\left(a_{* M, M, * M} \otimes \mathrm{id}_{M}\right) \circ a_{*^{*} M \otimes M,{ }^{*} M, M}^{-1}
$$

for the multiplication and $\operatorname{coev}_{M}^{\prime}(1)$ for the unit, and then transfering it to $\operatorname{End}(M)$ via the canonical linear isomorphism $\operatorname{End}(M) \simeq * M \otimes M$. Then a similar computation yields the following result.

Proposition 5.3: The H-module algebra structure induced on $\operatorname{End}(M)$ by the one of $* M \otimes M$ coincides with $\operatorname{End}(M)_{v}$. 
We give now an application of the identification $\operatorname{End}(M)^{v} \equiv \operatorname{end}(M)$. Let us first recall from Ref. 15 that a (left) Yetter-Drinfeld module over $H$ is a left $H$-module $M$, together with a left $H$-coaction on $M$ and some compatibility conditions between the two structures, ensuring that if we take the category of Yetter-Drinfeld modules, it is just the center of the monoidal category of left $H$-modules (we refer to Ref. 15 for detail).

Suppose now that $H$ is a quasi-Hopf algebra and $M$ is a finite dimensional Yetter-Drinfeld module. The following result generalizes the corresponding one for Hopf algebras proved in Ref. 8.

Proposition 5.4: $\operatorname{End}(M)^{v}$ and $\operatorname{End}(M)_{v}$ are algebras in the category of Yetter-Drinfeld modules over $H$.

Proof: We give the proof only for $\operatorname{End}(M)^{v}$, it is similar for $\operatorname{End}(M)_{v}$. It was proved in Ref. 4 that $M^{*}$, the left dual of $M$, becomes also a Yetter-Drinfeld module, so $M \otimes M^{*}$ is a YetterDrinfeld module too; by the identification $\operatorname{End}(M)^{v} \equiv M \otimes M^{*}$ via $\lambda$ we obtain that $\operatorname{End}(M)^{v}$ is also a Yetter-Drinfeld module. Since we already know that $\operatorname{End}(M)^{v}$ is an algebra in ${ }_{H} \mathcal{M}$, the only thing left to prove would be that the multiplication and unit of $\operatorname{End}(M)^{v}$ intertwine the corresponding $H$-coaction on $\operatorname{End}(M)^{v}$; a direct proof of these facts would be quite technical and difficult (even for the unit). However, we can give an immediate proof using the identification $\operatorname{End}(M)^{v}$ $\equiv \operatorname{end}(M)$. Namely, it was proved in Ref. 4 that $e v_{M}$ and $\operatorname{coev}_{M}$ are morphisms not only in ${ }_{H} \mathcal{M}$, but also in the Yetter-Drinfeld category, so the multiplication (5.5) of end $(M)$ is a morphism in the Yetter-Drinfeld category, and similarly for the unit of end $(M)$, which is exactly $\operatorname{coev}_{M}$, and we are done. Alternatively, we can obtain the result as a particular case of general results on braided tensor categories in Ref. 20.

\section{SOME EXAMPLES OF ENDOMORPHISM QUASIALGEBRAS: HEISENBERG DOUBLES}

We start this section by giving an example taken from Ref. 3, which will turn out to be of the type $\operatorname{End}(M)^{v}$. If $H$ is a finite dimensional quasi-Hopf algebra, in Ref. 3 was constructed the so-called quasi-smash product $H \overline{\#} H^{*}$, which is a left $H$-module algebra structure built on $H$ $\otimes H^{*}$, with multiplication, unit and $H$-action given, for all $h, h^{\prime} \in H$ and $\xi, \xi^{\prime} \in H^{*}$, by

$$
\begin{gathered}
(h \overline{\#} \xi)\left(h^{\prime} \overline{\#} \xi^{\prime}\right)=\sum h h_{1}^{\prime} x^{1} \overline{\#}\left(\xi \smile h_{2}^{\prime} x^{2}\right)\left(\xi^{\prime} \leftarrow x^{3}\right), \\
1_{H \overline{\#} H^{*}}=1_{H} \overline{\#} \varepsilon, \\
h \cdot\left(h^{\prime} \overline{\#} \xi\right)=h^{\prime} \overline{\#} h-\xi,
\end{gathered}
$$

where $\rightarrow$ and $\longleftarrow$ are the left and right regular actions of $H$ on $H^{*}$ given by

$$
(h-\xi)\left(h^{\prime}\right)=\xi\left(h^{\prime} h\right), \quad(\xi \leftarrow h)\left(h^{\prime}\right)=\xi\left(h h^{\prime}\right) .
$$

Then, in Ref. 3 was constructed a linear isomorphism,

$$
\mu: H \overline{\#} H^{*} \simeq \operatorname{End}(H), \mu(h \overline{\#} \xi)\left(h^{\prime}\right)=\sum \xi\left(h_{2}^{\prime} \tilde{p}^{2}\right) h h_{1}^{\prime} \tilde{p}^{1},
$$

for all $h, h^{\prime} \in H$ and $\xi \in H^{*}$, where $p_{L}=\Sigma \tilde{p}^{1} \otimes \tilde{p}^{2}=\Sigma X^{2} S^{-1}\left(X^{1} \beta\right) \otimes X^{3}$, with inverse

$$
\mu^{-1}: \operatorname{End}(H) \rightarrow H \overline{\#} H^{*}, \mu^{-1}(u)=\sum u\left(\widetilde{q}^{2}\left(e_{i}\right)_{2}\right) S^{-1}\left(\widetilde{q}^{1}\left(e_{i}\right)_{1}\right) \overline{\#} e^{i},
$$

for all $u \in \operatorname{End}(H)$, where $\left\{e_{i}\right\}$ and $\left\{e^{i}\right\}$ are dual bases in $H$ and $H^{*}$, and $q_{L}=\Sigma \widetilde{q}^{1} \otimes \widetilde{q}^{2}$ $=\Sigma S\left(x^{1}\right) \alpha x^{2} \otimes x^{3}$. Then, the $H$-module algebra structure of $H \overline{\#} H^{*}$ was transfered to $\operatorname{End}(H)$ via $\mu$, where it looks as follows (the multiplication, unit and $H$-action): 


$$
\begin{gathered}
\left(u_{-}^{\circ} u^{\prime}\right)(h)=\sum u\left(u^{\prime}\left(h x^{3} X_{2}^{3}\right) S^{-1}\left(S\left(x^{1} X^{2}\right) \alpha x^{2} X_{1}^{3}\right)\right) S^{-1}\left(X^{1}\right), \\
1_{\operatorname{End}(H)}=S^{-1}(\beta)-\mathrm{id}_{H},(h \cdot u)\left(h^{\prime}\right)=u\left(h^{\prime} h_{2}\right) S^{-1}\left(h_{1}\right),
\end{gathered}
$$

for all $u, u^{\prime} \in \operatorname{End}(H)$ and $h, h^{\prime} \in H$. Then one can easily check that this $H$-module algebra structure on $\operatorname{End}(H)$ is exactly $\operatorname{End}(M)^{v}$, where $M$ is $H$ regarded as a left $H$-module with action $h \cdot m=m S^{-1}(h)$ for all $m, h \in H$. The quasismash product $H \overline{\#} H^{*}$ was called the Heisenberg double of $H$ (its multiplication generalizes the one in the usual Heisenberg double of a Hopf algebra), cf. Ref. 3. For reasons to be discussed below, we call it the first Heisenberg double of $H$ and denote it by $\mathcal{H}_{1}(H)$.

To motivate this terminology, we discuss the case of the Heisenberg double $\mathcal{H}(H)$ of a finite dimensional Hopf algebra $H$. It is built on $H \otimes H^{*}$ and has the following tensor-categorical properties: it is a left $D(H)$-module algebra and a right $D(H)^{\text {cop }}$-module algebra [and hence a $D(H)$, $D(H)^{\text {cop }}$-bimodule algebra], where $D(H)$ is the Drinfeld double of $H$ and the actions of $D(H)$ on $\mathcal{H}(H)$ are the left and right regular actions if we regard $\mathcal{H}(H) \equiv D(H)^{*}$ as linear spaces, see Refs. 13 and 17 for all these. In particular, $\mathcal{H}(H)$ is a left $H$-module algebra [with action $h \cdot\left(h^{\prime} \# \xi\right)$ $\left.=h^{\prime} \# h-\xi\right]$ and a right $H^{\text {cop }}$-module algebra.

Now, if one tries to define a quasi-Hopf analogue of the Heisenberg double, one should obtain the same tensor-categorical properties as in the Hopf case. But since the three tensor-categorical properties of the Heisenberg double normally cannot be satisfied simultaneously in the quasi-Hopf case, it follows that one might be looking for (at least) three Heisenberg doubles of a finite dimensional quasi-Hopf algebra. The first one then should be $\mathcal{H}_{1}(H)=H \overline{\#} H^{*}$ defined in Ref. 3, which is a left $H$-module algebra, and a construction for the third was proposed in Ref. 17, providing a $D(H), D(H)^{\text {cop }}$-bimodule algebra. Now we propose a definition for the second one,

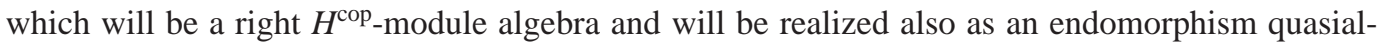
gebra.

Again we start with a general construction. If $H$ is a quasi-bialgebra and $A$ is a left $H$-module algebra with action $h \otimes a \mapsto h \cdot a$, then one can prove that $A^{\text {op }}$ becomes a right $H^{\text {op cop }}$-module algebra, with action $a \cdot h=h \cdot a$. Then we have the following result.

Proposition 6.1: Let $H$ be a quasi-Hopf algebra, $B$ an associative algebra and $v: H \rightarrow B$ an algebra map. Define a new multiplication on $B$ by

$$
b \odot b^{\prime}=\sum v\left(S^{-1}\left(x_{2}^{3} X^{3}\right)\right) b v\left(x_{1}^{3} X^{2} S^{-1}\left(x^{2} X^{1} \beta\right)\right) b^{\prime} v\left(x^{1}\right), \quad \forall b, b^{\prime} \in B,
$$

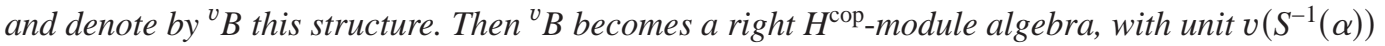
and right $\mathrm{H}$-action on ${ }^{v} \mathrm{~B}$ given by

$$
b_{v} \triangleleft h=\sum v\left(S^{-1}\left(h_{2}\right)\right) b v\left(h_{1}\right),
$$

for all $h \in H$ and $b \in{ }^{v} B$, where $\Delta(h)=\Sigma h_{1} \otimes h_{2}$ is the comultiplication of $H$.

Proof: Since $v$ is an algebra map, $v: H^{\mathrm{op}} \rightarrow B^{\mathrm{op}}$ is also an algebra map, which will be denoted by $v^{\text {op. }}$. Then we can consider $\left(B^{\text {op }}\right)^{\text {op }}$, which is a left $H^{\text {op }}$-module algebra, hence, by the above remark, we get that $\left(\left(B^{\mathrm{op}}\right)^{v^{\mathrm{op}}}\right)^{\text {op }}$ becomes a right $\left(H^{\mathrm{op}}\right)^{\mathrm{op} \text { cop }}=H^{\mathrm{cop}}$-module algebra, and one can easily see that ${ }^{v} B$ is exactly this $\left(\left(B^{\mathrm{op}}\right)^{v^{\mathrm{op}}}\right)^{\mathrm{op}}$.

In particular we may take $B=H, v=\mathrm{id}_{H}$, and obtain a right $H^{\text {cop }}$-module algebra structure on $H$, or we may take $M$ to be a left $H$-module and the map $v: H \rightarrow \operatorname{End}(M)$ as before, and we get a right $H^{\text {cop-module algebra }}{ }^{v} \operatorname{End}(M)$.

We are now interested in the case when $M=H$ with the left regular action on itself, so we have the algebra map $v: H \rightarrow \operatorname{End}(H), v(h)\left(h^{\prime}\right)=h h^{\prime}$, and we can consider the right $H^{\text {cop }}$-module algebra ${ }^{v} \operatorname{End}(H)$. Let us record that the right $H$-action on ${ }^{v} \operatorname{End}(H)$ is given by $\left(u_{v} \triangleleft h\right)\left(h^{\prime}\right)$ $=\Sigma S^{-1}\left(h_{2}\right) u\left(h_{1} h^{\prime}\right)$, for all $h, h^{\prime} \in H$ and $u \in{ }^{v} \operatorname{End}(H)$, where $\Delta(h)=\Sigma h_{1} \otimes h_{2}$ is the comultiplication 
of $H$. We propose this ${ }^{v} \operatorname{End}(H)$ as the second Heisenberg double of $H$ and denote it by $\mathcal{H}_{2}(H)$. This proposal will be supported by the next results.

Suppose now that $H$ is moreover finite dimensional. Then, besides the isomorphism $\mu$ introduced in Ref. 3, we can construct another linear isomorphism between $H \otimes H^{*}$ and $\operatorname{End}(H)$, namely,

$$
\nu: H \otimes H^{*} \rightarrow \operatorname{End}(H), \quad \nu(h \otimes \xi)\left(h^{\prime}\right)=\sum \xi\left(X^{2} h_{2}^{\prime} \widetilde{p}^{2}\right) S^{-1}\left(X^{3}\right) h X^{1} h_{1}^{\prime} \tilde{p}^{1},
$$

for all $h, h^{\prime} \in H$ and $\xi \in H^{*}$; one can prove as in Ref. 3 that its inverse is given by

$$
\nu^{-1}: \operatorname{End}(H) \rightarrow H \otimes H^{*}, \quad \nu^{-1}(u)=\sum S^{-1}\left(x^{3}\right) u\left(\widetilde{q}^{2}\left(e_{i}\right)_{2}\right) S^{-1}\left(\widetilde{q}^{1}\left(e_{i}\right)_{1}\right) x^{1} \otimes e^{i} \leftarrow x^{2},
$$

for all $u \in \operatorname{End}(H)$.

Now, we consider on $\operatorname{End}(H)$ the right $H^{\text {cop }}$-module algebra structure ${ }^{v} \operatorname{End}(H)$, and we can transfer it to $H \otimes H^{*}$ via the isomorphisms $\mu$ or $\nu$, thus obtaining two (isomorphic) right $H^{\mathrm{cop}}$-module algebra structures on $H \otimes H^{*}$.

Proposition 6.2: If we denote by $\hookleftarrow$, respectively $\leftarrow$, the right $H$-module structures of $H$ $\otimes H^{*}$ obtained by transfer via $\mu$, respectively $\nu$, then $\hookleftarrow$ and $\leftarrow$ look as follows:

$$
\begin{aligned}
& \left(h^{\prime} \otimes \xi\right) \hookleftarrow h=\sum S^{-1}\left(h_{2}\right) h^{\prime} h_{(1,1)} \otimes \xi \leftarrow h_{(1,2)}, \\
& \left(h^{\prime} \otimes \xi\right)-h=\sum S^{-1}\left(h_{(2,2)}\right) h^{\prime} h_{1} \otimes \xi \leftarrow h_{(2,1)},
\end{aligned}
$$

for all $h, h^{\prime} \in H$ and $\xi \in H^{*}$, where $\Delta(h)=\sum h_{1} \otimes h_{2}$ is the comultiplication of $H$.

Proof: We give the proof only for $\hookleftarrow$, the one for - is similar. We compute $\left(h^{\prime} \otimes \xi\right) \hookleftarrow h$ $\in H \otimes H^{*}$ by applying it to an element $g \in H$ on the second component. We have

$$
\begin{aligned}
\left(\left(h^{\prime} \otimes \xi\right) \hookleftarrow h\right)(g) & =\mu^{-1}\left(\mu\left(h^{\prime} \otimes \xi\right)_{v} \triangleleft h\right)(g) \\
& =\sum\left(\mu\left(h^{\prime} \otimes \xi\right)_{v} \triangleleft h\right)\left(\widetilde{q}^{2} g_{2}\right) S^{-1}\left(\widetilde{q}^{1} g_{1}\right) \\
& =\sum\left(v\left(S^{-1}\left(h_{2}\right)\right) \mu\left(h^{\prime} \otimes \xi\right) v\left(h_{1}\right)\right)\left(\widetilde{q}^{2} g_{2}\right) S^{-1}\left(\widetilde{q}^{1} g_{1}\right) \\
& =\sum S^{-1}\left(h_{2}\right) \mu\left(h^{\prime} \otimes \xi\right)\left(h_{1} \widetilde{q}^{2} g_{2}\right) S^{-1}\left(\widetilde{q}^{1} g_{1}\right) \\
& =\sum S^{-1}\left(h_{2}\right) \xi\left(h_{(1,2)} \widetilde{q}_{2}^{2} g_{(2,2)} \widetilde{p}^{2}\right) h^{\prime} h_{(1,1)} \widetilde{q}_{1}^{2} g_{(2,1)} \widetilde{p}^{1} S^{-1}\left(g_{1}\right) S^{-1}\left(\widetilde{q}^{1}\right) \\
(2.22) & =\sum \xi\left(h_{(1,2)} \widetilde{q}_{2}^{2} \widetilde{p}^{2} g\right) S^{-1}\left(h_{2}\right) h^{\prime} h_{(1,1)} \widetilde{q}_{1}^{2} \widetilde{p}^{1} S^{-1}\left(\widetilde{q}^{1}\right) \\
(2.20) & =\sum \xi\left(h_{(1,2)} g\right) S^{-1}\left(h_{2}\right) h^{\prime} h_{(1,1)}=\sum\left(S^{-1}\left(h_{2}\right) h^{\prime} h_{(1,1)} \otimes \xi \leftarrow h_{(1,2)}\right)(g),
\end{aligned}
$$

and the proof is finished.

Now, let $H$ be a finite dimensional quasi-Hopf algebra. We recall from Refs. 10 and 11 the two realizations of the quantum double of $H$ built on $H^{*} \otimes H$, hereafter denoted by $D_{1}(H)$ and $D_{2}(H)$, whose multiplications are, respectively, given, for all $\xi, \xi^{\prime} \in H^{*}$ and $h, h^{\prime} \in H$, by

$$
\begin{aligned}
& (\xi \otimes h)\left(\xi^{\prime} \otimes h^{\prime}\right)=\sum\left(\Omega^{1}-\xi \leftarrow \Omega^{5}\right)\left(\Omega^{2} h_{(1,1)}-\xi^{\prime} \leftarrow S^{-1}\left(h_{2}\right) \Omega^{4}\right) \otimes \Omega^{3} h_{(1,2)} h^{\prime}, \\
& (\xi \otimes h)\left(\xi^{\prime} \otimes h^{\prime}\right)=\sum\left(\omega^{1}-\xi \leftarrow \omega^{5}\right)\left(\omega^{2} h_{1}-\xi^{\prime} \leftarrow S^{-1}\left(h_{(2,2)}\right) \omega^{4}\right) \otimes \omega^{3} h_{(2,1)} h^{\prime},
\end{aligned}
$$

where $\Omega=\Sigma \Omega^{1} \otimes \cdots \otimes \Omega^{5}, \omega=\Sigma \omega^{1} \otimes \cdots \otimes \omega^{5} \in H^{\otimes 5}$ are given by

$$
\Omega=\sum X_{(1,1)}^{1} x^{1} y^{1} \otimes X_{(1,2)}^{1} x^{2} y_{1}^{2} \otimes X_{2}^{1} x^{3} y_{2}^{2} \otimes S^{-1}\left(f^{1} X^{2} y^{3}\right) \otimes S^{-1}\left(f^{2} X^{3}\right),
$$




$$
\omega=\sum x^{1} \otimes x^{2} Y^{1} \otimes x_{1}^{3} X^{1} Y_{1}^{2} \otimes S^{-1}\left(f^{1} x_{(2,1)}^{3} X^{2} Y_{2}^{2}\right) \otimes S^{-1}\left(f^{2} x_{(2,2)}^{3} X^{3} Y^{3}\right),
$$

and where $f=\Sigma f^{1} \otimes f^{2}$ is the twist defined in (2.15).

Using these formulas, one can prove that the comultiplications of $D_{1}(H)^{*}$ and $D_{2}(H)^{*}$ (both identified with $H \otimes H^{*}$ as linear spaces), dual to the above multiplications, are given by

$$
\begin{aligned}
& \Delta_{D_{1}(H)^{*}}(h \otimes \xi)=\sum\left(\Omega^{5} h_{1} \Omega^{1} \otimes\left[e^{i}\left(\xi_{1} \leftarrow \Omega^{3}\right)\right] e^{j}\right) \otimes\left(S^{-1}\left(e_{j}\right) \Omega^{4} h_{2} \Omega^{2} e_{i} \otimes \xi_{2}\right), \\
& \Delta_{D_{2}(H)^{*}}(h \otimes \xi)=\sum\left(\omega^{5} h_{1} \omega^{1} \otimes e^{i}\left[\left(\xi_{1} \leftarrow \omega^{3}\right) e^{j}\right]\right) \otimes\left(S^{-1}\left(e_{j}\right) \omega^{4} h_{2} \omega^{2} e_{i} \otimes \xi_{2}\right),
\end{aligned}
$$

for all $h \in H$ and $\xi \in H^{*}$, where $\Delta(\xi)=\Sigma \xi_{1} \otimes \xi_{2}$ is the comultiplication of $H^{*}$ and $\left\{e_{i}\right\}$ and $\left\{e^{i}\right\}$ are dual bases in $H$ and $H^{*}$. Using these formulas, one can prove that the right regular actions of $D_{1}(H)$ on $D_{1}(H)^{*}$ and of $D_{2}(H)$ on $D_{2}(H)^{*}$, respectively, look as follows:

$$
\begin{gathered}
\left(h^{\prime} \otimes \xi\right) \leftarrow\left(\xi^{\prime} \otimes h\right)=\sum \xi^{\prime}\left(\Omega^{5} h_{1}^{\prime} \Omega^{1}\right) S^{-1}\left(h_{2}\right) \Omega^{4} h_{2}^{\prime} \Omega^{2} h_{(1,1)} \otimes \xi \sqcup \Omega^{3} h_{(1,2)}, \\
\left(h^{\prime} \otimes \xi\right) \leftarrow\left(\xi^{\prime} \otimes h\right)=\sum \xi^{\prime}\left(\omega^{5} h_{1}^{\prime} \omega^{1}\right) S^{-1}\left(h_{(2,2)}\right) \omega^{4} h_{2}^{\prime} \omega^{2} h_{1} \otimes \xi \leftarrow \omega^{3} h_{(2,1)},
\end{gathered}
$$

for all $h, h^{\prime} \in H$ and $\xi, \xi^{\prime} \in H^{*}$. By taking $\xi^{\prime}=\varepsilon$ in these formulas, we get two right $H$-module structures on $H \otimes H^{*}$, and one can easily see that they are exactly (6.11) and (6.12).

Let us mention that, if the proposed definitions of $\mathcal{H}_{1}(H)$ and $\mathcal{H}_{2}(H)$ are natural, they should be not only left $H$ and, respectively, right $H^{\text {cop }}$-module algebras, but also left $D(H)$ and, respectively, right $D(H)^{\text {cop }}$-module algebras, but so far we have not been able to prove this (direct computations are very cumbersome).

\section{ACKNOWLEDGMENTS}

Research supported by the bilateral project "Hopf Algebras in Algebra, Topology, Geometry and Physics" of the Flemish and Romanian Ministries of Research. Parts of this paper have been written while the first author (F.P.) was visiting the University of Antwerp, and he would like to thank UA for its warm hospitality. F.P. was also partially supported by the programmes SCOPES and EURROMMAT.

\footnotetext{
${ }^{1}$ Albuquerque, H. and Majid, S., "Quasialgebra structure of the octonions," J. Algebra 220, 188-224 (1999).

${ }^{2}$ Baez, J. C., "The octonions," Bull., New Ser., Am. Math. Soc. 39, 145-205 (2002).

${ }^{3}$ Bulacu, D. and Caenepeel, S., "Two-sided two-cosided Hopf modules and Doi-Hopf modules for quasi-Hopf algebras," J. Algebra 270, 55-95 (2003).

${ }^{4}$ Bulacu, D., Caenepeel, S., and Panaite, F., "Yetter-Drinfeld categories for quasi-Hopf algebras," math.QA/0311379.

${ }^{5}$ Bulacu, D. and Nauwelaerts, E., "Relative Hopf modules for (dual) quasi-Hopf algebras," J. Algebra 229, 632-659 (2000).

${ }^{6}$ Bulacu, D., Panaite, F., and Van Oystaeyen, F., "Quasi-Hopf algebra actions and smash products," Commun. Algebra 28, 631-651 (2000).

${ }^{7}$ Bulacu, D., Panaite, F., and Van Oystaeyen, F., "Quantum traces and quantum dimensions for quasi-Hopf algebras," Commun. Algebra 27, 6103-6122 (1999).

${ }^{8}$ Caenepeel, S., Van Oystaeyen, F., and Zhang, Y., "Quantum Yang-Baxter module algebras," K-Theory 8, 231-255 (1994).

${ }^{9}$ Drinfeld, V. G., Quasi-Hopf algebras, Leningrad Math. J. 1, 1419-1457 (1990).

${ }^{10}$ Hausser, F. and Nill, F., "Diagonal crossed products by duals of quasi-quantum groups," Rev. Math. Phys. 11, 553-629 (1999).

${ }^{11}$ Hausser, F. and Nill, F., "Doubles of quasi-quantum groups," Commun. Math. Phys. 199, 547-589 (1999).

${ }^{12}$ Kassel, C., "Quantum groups," Graduate Texts in Mathematics (Springer-Verlag, Berlin, 1995), Vol. 155.

${ }^{13}$ Lu, J.-H., "On the Drinfeld double and the Heisenberg double of a Hopf algebra," Duke Math. J. 74, 763-776 (1994).

${ }^{14}$ Majid, S., Foundations of Quantum Group Theory (Cambridge University Press, Cambridge, 1995).

${ }^{15}$ Majid, S., "Quantum double for quasi-Hopf algebras," Lett. Math. Phys. 45, 1-9 (1998).

${ }^{16}$ Montgomery, S., "Hopf algebras and their actions on rings," CBMS Regional Conference Series, Vol. 82 (American Mathematical Society, Providence, RI, 1993).

${ }^{17}$ Panaite, F., "Doubles of (quasi) Hopf algebras and some examples of quantum groupoids and vertex groups related to them," math.QA/0101039.
} 
${ }^{18}$ Schafer, R. D., An Introduction to Nonassociative Algebras (Academic, New York, 1966).

${ }^{19}$ Sweedler, M. E., Hopf Algebras (Benjamin, New York, 1969).

${ }^{20}$ Van Oystaeyen, F. and Zhang, Y., "The Brauer group of a braided monoidal category," J. Algebra 202, 96-128 (1998). 